\title{
Sequential SEM imaging of microbial calcite precipitation consolidation treatment
}

\author{
Nadim C. Scherrer ${ }^{\mathrm{a}}{ }^{\mathbb{D}}$, Matthias Kocsis, Petra Dariz, Claire Gervais \\ HKB, Bern University of Applied Sciences, Fellerstrasse 11, 3027 Bern, Switzerland
}

Received: 5 November 2020 / Accepted: 25 April 2021

(C) The Author(s) 2021

\begin{abstract}
Cultural heritage built from limestone is prone to deterioration by chemical weathering, a natural process, that is enhanced by pollution. There are many historic monuments built from calcareous rocks that suffer from deterioration, and thus there have been a number of approaches over the last few decades to consolidate these types of rocks and surfaces. Using natural biological processes by fostering the activity of calcite-producing bacteria, also referred to as biomineralization, is one strategy that has also been commercialized. The base of proving the effectiveness of any surface treatment is the observation of the surface at sequential stages before and after treatment, as well as after exposure to weathering. Due to the heterogeneity of natural materials and processes, our aim was to observe identical test areas at the micron scale throughout the observation period. In order to achieve this on a tungsten SEM, we employed a beam deceleration accessory that allowed low $\mathrm{kV}$ imaging on non-conductive surfaces at a sufficiently high image resolution with a modified sample holder accommodating drill cores of $25 \mathrm{~mm}$ diameter and up to $15 \mathrm{~mm}$ height. The presented method is capable of producing time-sequenced images on the same test area on natural rock surface samples without manipulation for imaging purposes. This offers interesting perspectives for effective documentation of such processes in various fields.
\end{abstract}

\section{Introduction}

Cultural heritage built from limestone is prone to deterioration by chemical weathering, a natural process, that is enhanced by pollution. The abundance of calcareous rocks means that there are many historic monuments throughout Europe requiring conservation measures for their preservation. The idea to consolidate these monuments by biological processes with the help of calcite-producing bacteria [1] seems an obvious approach that has been explored beyond the last two decades by several research groups [2-7]. Meanwhile there are commercial products available making use of this natural process of microbial calcite formation, following different strategies. Nature, however, is a complex interactive system with many factors at various scales influencing whether the treatment ends as a success or failure. The combination of rock type, porosity, climatic region, weather, exposure, bacterial type, nutrient, etc., leads to variable environments that need to be accounted for in order to foster the intended bacterial activity and, avoid unwanted side effects. At the other end, when

\footnotetext{
a e-mail: nadim.scherrer@hkb.bfh.ch (corresponding author)
} 
it comes to on-site large-scale application beyond the laboratory experiment, there are many other factors deciding on viability and success.

Visualization of the effects of a treatment is the key to understand processes and not the least to convince whether theory meets the real world. The scanning electron microscope (SEM) is a widely accessible tool to look at the micro- and nanoscale of all types of surfaces. With respect to documentation of the effect of bio-mineralization treatments, this is the technique research groups apply to visualize what is being produced on the substrates chosen [3, 5-10]. Within the published articles, samples, however, were always coated to achieve conductivity-and, as a consequence, observations were never made on the same sample at the identical location throughout the process. This, in part, is likely due to the difficulty of imaging non-conductive samples at sufficiently high quality with minimal penetration depth (low $\mathrm{kV}$ ), which, essentially is required to account for the sensitivity of organic material to the electron beam and the thinness of the newly formed material. The key would thus be to be able to observe non-conductive surfaces of rock samples at low acceleration voltage with reproducible positioning. Here we present a strategy that is capable of delivering SEM images at various (dry) stages of the process-original (cleaned) surface, after the consolidation treatment, and after outdoor weathering - at the same location with a standard tungsten cathode variable pressure VP-SEM and a beam deceleration accessory.

Beam deceleration (BD) reduces the incoming electron beam energy by applying a bias to the sample, while accelerating the outgoing signal again toward the detector. This improves resolution and contrast at low $\mathrm{kV}$ imaging with reduced penetration depth, and results in more surface detail [11]. While best results are achieved with conductive samples, it is possible to image non-conductive and heat-sensitive surfaces since using minimal landing energies of 0.5 to $4 \mathrm{kV}$. Publications referring to the beam deceleration technique generally dealt with thin and flat sections [11-15].

\section{Materials and methods}

The rock types selected as substrates for these microbial calcite precipitation tests [16] were limestones with high porosity: Barrois-Oolith from Savonnières-en-Perthois, Meuse, France, and Muschelkalk from Wuerenlos, Aargau, Switzerland. Both have experienced high popularity as a source material for buildings and sculptures north of the alps and have been used since roman times. The focus in this paper was limited to the former type.

The microbial treatment relied on the commercially available product KBYO M-3P (kbyobiological.com) which was applied by spraying the surface of multiple drill cores of $25 \mathrm{~mm}$ diameter, according to instructions supplied by kbyobiological.com, including reapplication within the first 5-7 days and conditioning within the first month $\left(\sim 20^{\circ} \mathrm{C}\right.$ ambient temperature and $\sim 65-55 \% \mathrm{rH}$ ).

Surface VIS imaging was performed using a Leica S6D stereomicroscope and a Keyence VHX1000 digital microscope, equipped with the VH-Z20R objective. These images were produced to have a visual overview and colour control of the different sample surfaces, as well as positioning control within the SEM. Surface topographic and compositional contrast images were generated on a standard Zeiss EVO MA10 tungsten emission scanning electron microscope (SEM) equipped with variable pressure (VP) air admittance, a 5 segment HDBSD backscattered electron detector (BSE) and a beam deceleration (BD) kit to put a bias voltage on a single sample holder. SEM imaging was performed at 4 to $5 \mathrm{kV}$ acceleration voltage, 50 to $70 \mathrm{pA}$ beam current, beam deceleration volts between 3500 and $4000 \mathrm{~V}$ (which equals to a landing energy of 1 to $1.5 \mathrm{kV}$ ), all performed in high vacuum mode on non-coated 

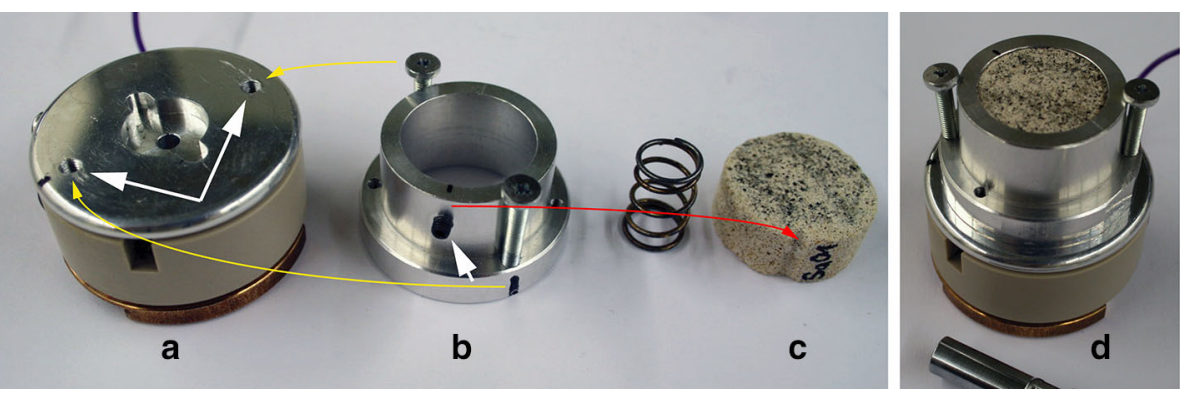

Fig. 1 Modified stage bias single-stub $(12 \mathrm{~mm})$ accessory with a additionally drilled and threaded holes, b aluminum socket with top lid and added grub screw to accommodate 25-mm-diameter rock samples of up to $15 \mathrm{~mm}$ thickness. d Fully assembled setup. With the aluminum socket mounted, the field lines emerge from the top lid for effective beam deceleration on the sample surface

surfaces. Images for comparison with standard imaging conditions were acquired as stated in the captions.

As the stage bias single stub accessory (Fig. 1a) is designed for thin and flat samples such as e.g. microtome sections of biological tissues, e.g. [12], some amendments were necessary to accommodate rock samples of $25 \mathrm{~mm}$ diameter and up to $15 \mathrm{~mm}$ height. The sample holder was modified (Fig. 1) by adding two threaded holes to accommodate the aluminum socket of a previous sample holder for cylindrical geological sections of $25.4 \mathrm{~mm}$ diameter. A grub screw was added on the side to mount the cylindrical samples with a notch in reproducible orientation. A spring is used to push the sample toward the top lid of the socket in order to assure optimum contact to the conducting lid and that most of the rough surface is at a similar level as the aluminum lid, from where the beam deceleration field lines emerge.

To account for the surface topography and the limited depth of field, image sequences were acquired manually at multifocal levels. These were then compiled into a single image using the software Heliconfocus Vers. 7 (heliconsoft.com), rendering with the method C (pyramid).

\section{Results and discussion}

\subsection{General technical aspects}

On natural, rough and heterogenous surfaces, effective and true comparison of process related changes is by observing the same area through the stages of the process. This means that surface imaging of such non-conductive surfaces in original state, after treatment and later after exposure must be possible at sufficiently resolved quality without applying coatings for imaging purposes. Using the variable pressure mode (VP-SEM) at 30-50 Pa in combination with the BSE detector requires settings, that do not deliver the sensitivity required to image thin and organic structures with sufficient quality. A significant disadvantage is the necessity of an aperture between chamber and gun to account for the variable pressure and as such reducing the field of view and signal intensity. Beam deceleration, on the other hand, is performed in high vacuum mode and thus does not require the reducing aperture in the beam path. The weak landing energy by using beam deceleration results in strongly reduced penetration depth and beam spot size, leading to much improved surface sensitivity and surface definition of very thin organic structures such as cell membranes (Fig. 2), while 

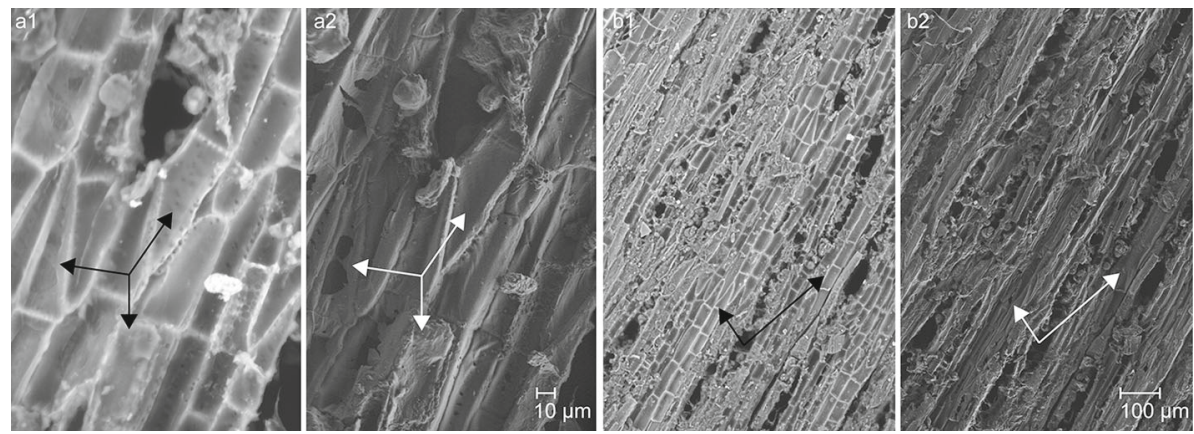

Fig. 2 SEM-BSE images of uncoated wood-cell structures of lindenbast. a1 and b1 were acquired in low vacuum $(40 \mathrm{~Pa})$ at $20 \mathrm{kV}$ and $100 \mathrm{pA}$. a2 and b2 were generated in high vacuum mode at $5 \mathrm{kV}, 70 \mathrm{pA}$ and $4000 \mathrm{~V}$ beam deceleration ( $1 \mathrm{kV}$ landing energy). Series A (a1 vs. a2) focuses on the low penetration and improved surface detail on very thin organic structures such as cell membranes using beam deceleration (a2) on non-coated and non-conductive surfaces. When the structure rather than the surface of biological organisms is of interest (series $\mathrm{B}$ ), then the penetrating power of the higher $\mathrm{kV}$ is capable of delivering an X-ray-like image of the structure, in this case emphasizing the cell walls (higher density of organic material) and strongly improving the recognition of the cell pattern (b1 vs. b2)
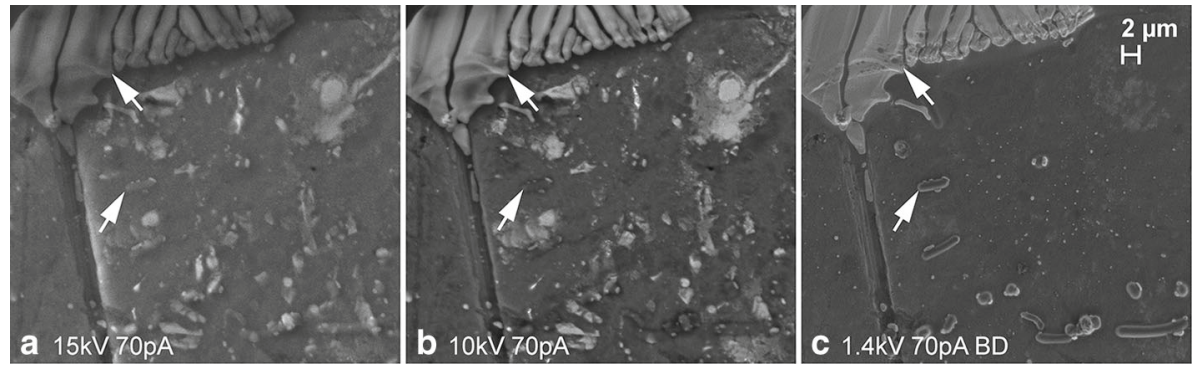

Fig. 3 SEM-BSE images on carbon-coated samples of a dried drop of KBYO nutrient solution on a glass slide after interaction with an immersed rock sample. The image series compares surface sensitivity at a $15 \mathrm{kV}$, b $10 \mathrm{kV}$ and at c $5 \mathrm{kV}$ with $3600 \mathrm{~V}$ beam deceleration (i.e., $1.4 \mathrm{kV}$ landing energy). Arrows point at surface details and at bacteria on the surface, which are best imaged and recognized using beam deceleration (c)

still giving some density contrast of thin, organic materials sitting on top of inorganic substrates (Figs. 3 and 4) using a backscattered electron detector. While a backscattered electron detector generally suffers from very weak signal efficiency at low $\mathrm{kV}$, it is less affected by charging than a secondary electron detector. Combined with the beam deceleration accessory, signal efficiency and contrast of the backscattered image are significantly improved. Thus, the backscattered electron signal delivers an image within reasonable acquisition times ( 1 to 3 min. per focus level) and a density contrast even at low landing energies, giving the advantage of recognizing, e.g., very thin organic biofilms, bacteria and newly formed microbial calcite on non-coated rock samples (Figs. 4 and 5). Since there is no need for a coating with carbon or gold to achieve stable SEM images with this strategy, it is possible to observe the identical field of view at various stages of interest, be it days, weeks, months or years apart (Figs. 5, 6, and 7). Limitation in the depth of focus is successfully addressed by acquiring multiple images at sequential focal levels and compiling them to a multifocal image. 


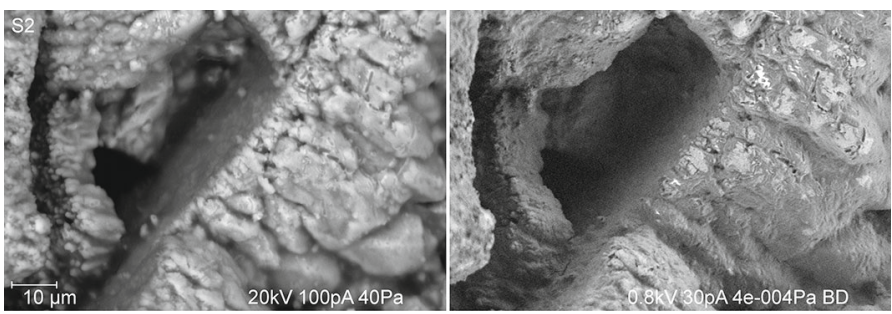

Fig. 4 Comparison of imaging at $40 \mathrm{~Pa}$ chamber pressure (low vacuum) and $20 \mathrm{kV}$ (left) versus high vacuum mode and $3 \mathrm{kV}$ acceleration voltage with $2200 \mathrm{~V}$ beam deceleration $(0.8 \mathrm{kV}$ landing energy) on a nonconductive and charging surface (right). The biofilm formation of EPS encapsulating emerging bacteria upon KBYO nutrient application and conditioning, is only visible in the righthand image as a dark grey cover (organic) on the lighter grey calcite substrate (higher-density rock)

\subsection{Imaging stages of artificially induced microbial calcite formation and weathering}

The sequential images achieved herewith at the identical location on various test samples give a different insight to the changes induced by the treatment. Imaging at the early stages after treatment and conditioning clearly shows the initial formation of a biofilm of extracellular polymeric substance (EPS), more or less encapsulating large numbers of bacteria emerging from the depth of the porous rock to the surface (Fig. 5a). Imaging the identical location 3 months later upon outdoor weathering (Fig. 5b) unveils complete retreat of the EPS biofilm and bacteria, and the emergence of a newly formed microbial calcite crust. While this crust is reconnecting neighboring grains, it does not-visually-grow to a closed film that could clog the original surface. The open 'karst'-like structure leads, however, to a considerable increase in surface area. Comparing the situation 13 months later (Fig. 5c) reveals that under the prevalent climatic conditions of the experiment, bacterial activity had ceased and weathering lead to renewed erosion of the microbial calcite at the exposed surface.

A new set of experiments (Figs. 6 and 7) replicating the initial series (Figs. 4 and 5) was run in order to include beam deceleration imaging of the pretreatment state as a reference with identical imaging settings. This was contemplated as necessary since pre-existing organic material or other biological growth (dark grey material in Fig. 6a1 and a2) may be present. The chosen substrate, KBYO nutrient application and procedure were identical. Figure 6 represents such a series with the clean, pretreatment state (a), versus the activated state after the treatment (b) with bacteria dispersed across the surface and initiation of microbial calcite deposition, as well as the situation 5 months later after exposure to outdoor weathering (c). With the intent to apply a consolidating treatment, Fig. 6b clearly demonstrates that the microbial activity does have the potential to reconnect grains with bio-calcite bridges, and that these may survive (Fig. 6c) the somewhat harsher climatic conditions prevalent in northern Switzerland. What these images further tell, however, is that there was no further activity upon completion of the conditioning period in this second series, which never reached the comparatively impressive productivity of the first series (Fig. 5). Putting this into perspective with the heterogeneous nature of the rock surface, the image series in Fig. 7 (compare images a3 versus b3 at arrow) demonstrates that the bacterial activity seems to occur in clusters, with some locations of increased microbial calcite production (b3) and other areas with only marginal activity (b2). At this stage, it is not clear which criteria lead to preferential sites for activity. Comparing Fig. $7 \mathrm{~b} 3$ with Fig. 5b further unveils that slightly varying conditions may result in different morphologies, which in turn results in variable stability, when it comes to weathering and erosion (Fig. 7c vs. Fig. 5c). 

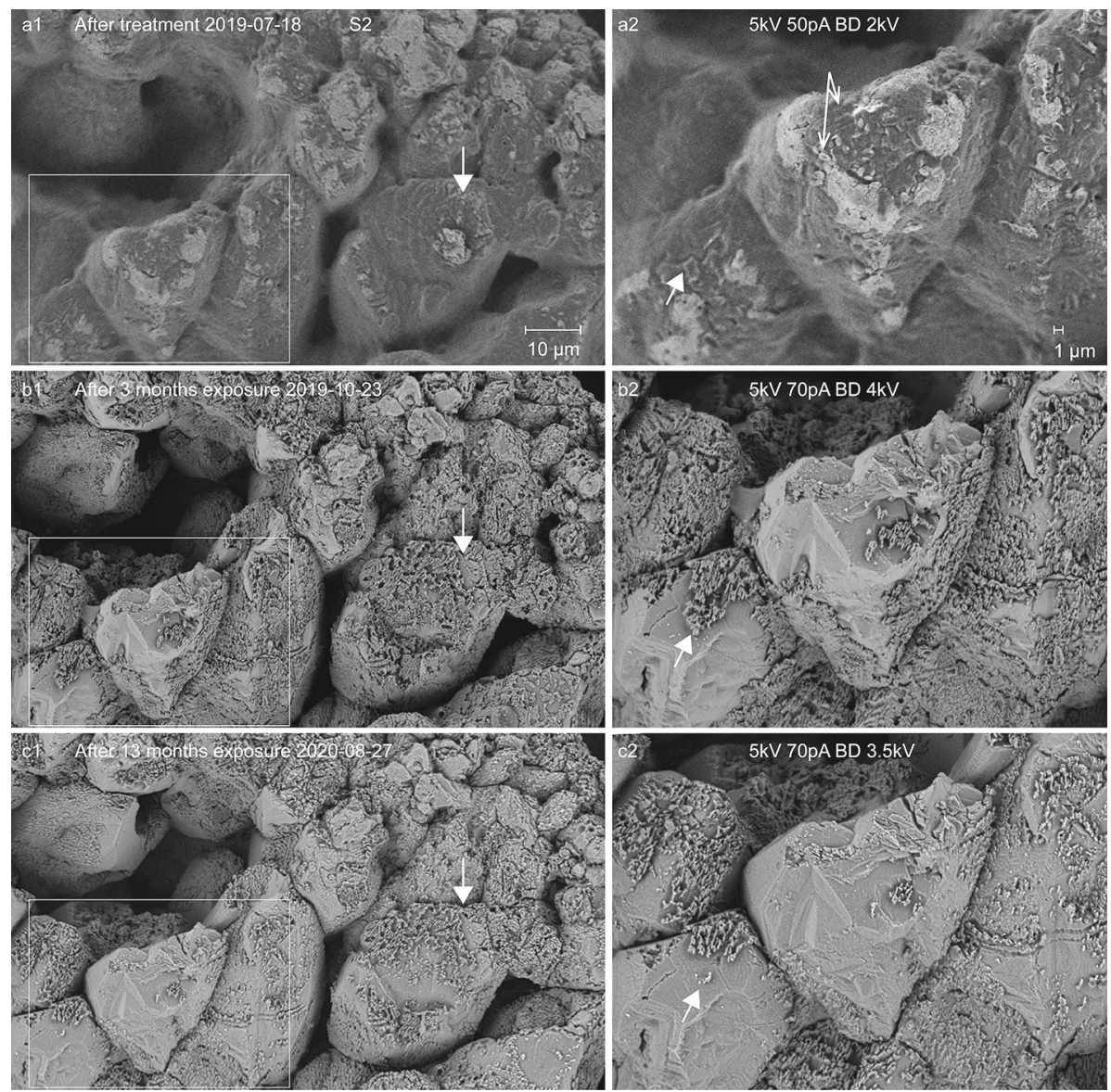

Fig. 5 SEM-BSE image sequence produced with the beam deceleration accessory on a non-conductive rock sample surface (Savonnières) at the identical locality at three different stages (months apart) of the microbial calcite formation and weathering process. a upon completion of the KBYO-treatment and conditioning over 1 month; b 3 months later after outdoor exposure (rooftop, $45^{\circ}$ inclination, SSE aspect, $47.5^{\circ}$ latitude); c 13 months later after additional outdoor weathering. Simple arrows in a2) point at mobilization of bacteria (oval body) from the inside of the rock sample to the surface upon application of the KBYO nutrient (dark, organic layer represents biofilm of EPS) within the 30 days of conditioning. The open arrow in b1) points at a cavity within the microbial crust, following the death of the organism. Filled arrows (sequence a-c) point at the development (b) and beginning, renewed erosion of the microbial calcite crust (c) within a year of exposure to weathering in this climatic environment $\left(450 \mathrm{~m}\right.$ a.s.1., yearly avg. $\mathrm{T} 9.2{ }^{\circ} \mathrm{C}$, min. avg. January $0^{\circ}$, max. avg. July $18.2^{\circ} \mathrm{C}$; annual rainfall $1049 \mathrm{~mm}$ ). Images $\mathbf{b}$ and $\mathbf{c}$ are multifocal compilations of 3-4 focal levels (greater depth of focus)

The advantage of imaging identical locations through multiple stages of a process are obvious: There is no speculation on how to translate the situation from one sample to another. An image series that can be superimposed undoubtedly documents changes that have taken place, telling a comprehensible story. Characterizing the results of the presented case study on microbial calcite growth and weathering, it appears that a successful procedure involving KBYO may require prolonged treatment and conditioning to ensure sufficient microbial calcite build-up in this climatic region. As such, the process can be regarded as limited in 

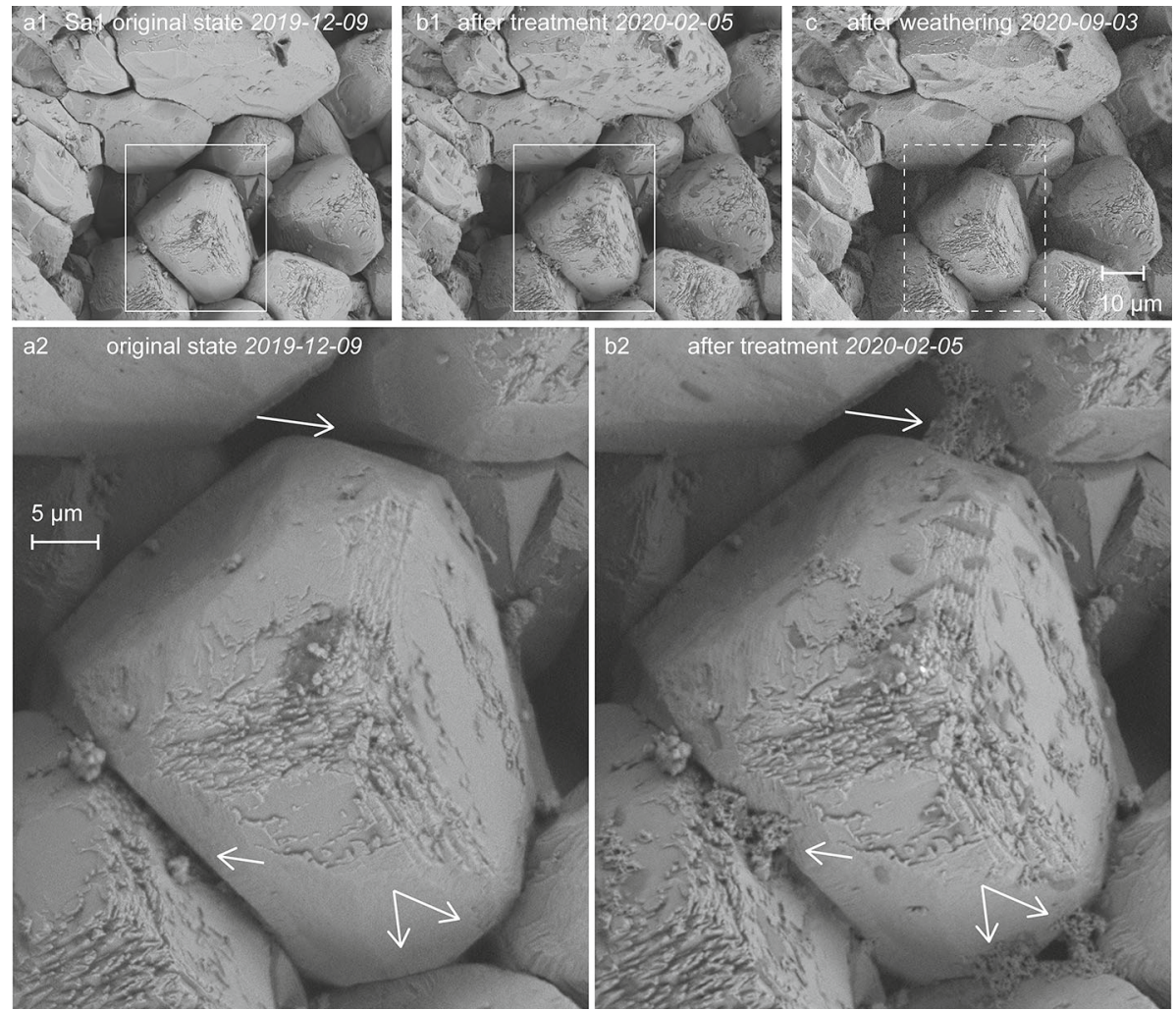

Fig. 6 SEM-BSE image sequence acquired with beam deceleration on a second and later series of Savonnière samples. These samples were imaged with identical conditions ( $5 \mathrm{kV} 70 \mathrm{pA}$ BD4000V), including the pretreatment condition: a original state before the treatment, $\mathbf{b} \sim 1$ week after the 30-day KBYO-treatment with conditioning, $\mathbf{c} 7$ months later with 3 months of outdoor exposure to weathering as in (Fig. 5). Darker grey elongate dots in (b) are interpreted as activated bacteria, not visible in (a) and neither (c). Arrows in a2 versus b2 point at microbial calcite formation, bridging the interstices between neighboring calcite grains and as such initiating a consolidating effect. These bridges are still recognizable in $\mathrm{c} 1$ upon weathering, though already slightly reduced. Compared to Fig. 5, conditions obviously did not lead to a similar microbial crust formation

the sense that it is nutrient and climatic conditioning controlled. Exposing the delicate, high surface morphology of the newly formed microbial calcite to local weathering conditions lead to rapid erosion at exposed surfaces (Figs. 5, 6, and 7). Nevertheless, the rather open structure, combined with bacterial mobility, constitute considerable advantages for potential sub-surface consolidation. In other words, the treatment is likely to cause minimal impediment on diffusive properties of the rock, while potentially reaching greater depths than purely chemical treatments. Direct documentation of the process in a similar way below the surface, i.e., in a depth profile or transect will require yet a different approach. Nevertheless, by training the eye what is being observed at the identical site through the process at the surface may be better translated to observations made on separate sections. 


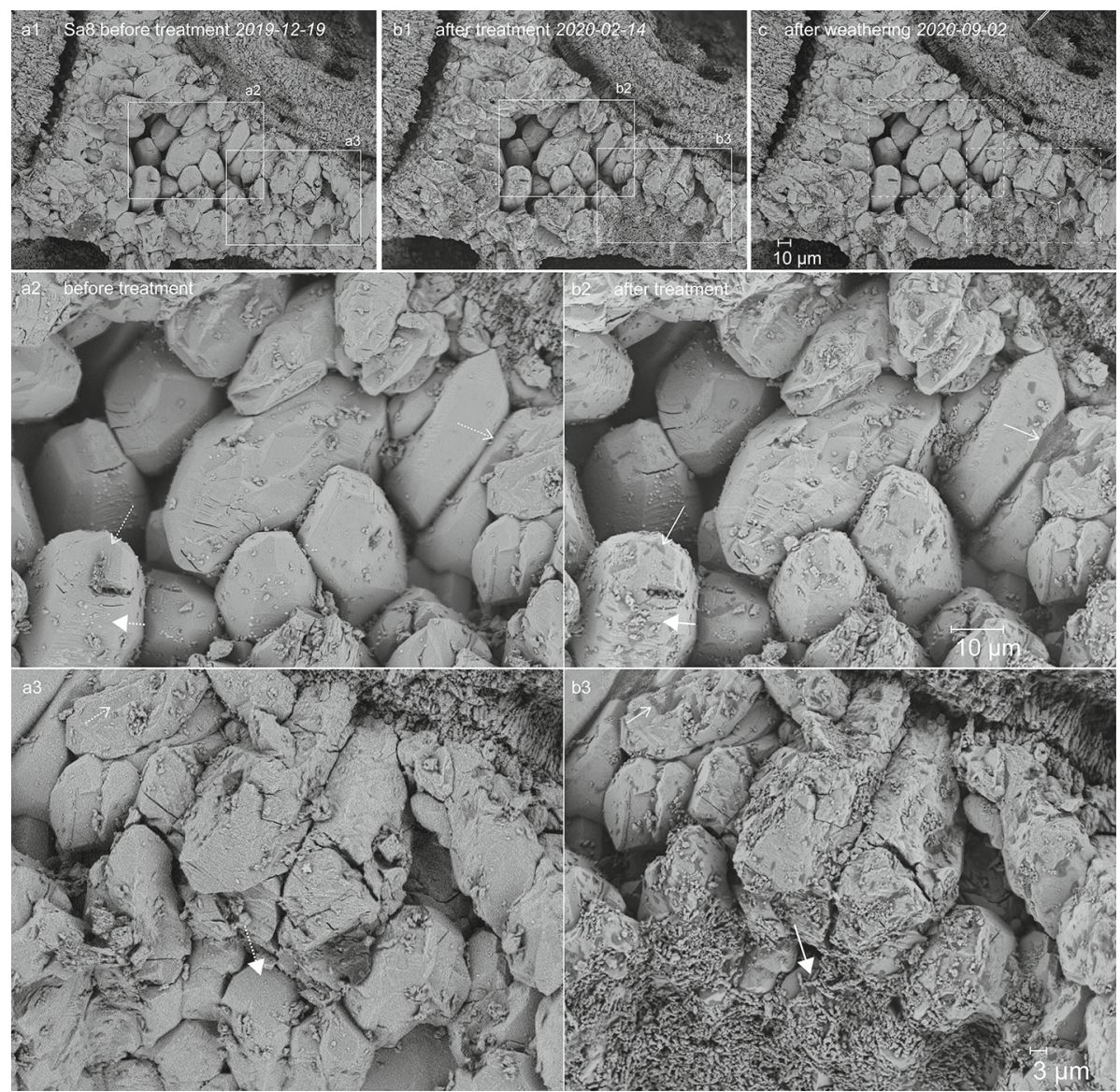

Fig. 7 Another Savonnières sample of the later treatment series imaged with beam deceleration before (a), after KBYO-treatment (b) and after a short exposure period (c) of 3 months (June-August 2020) at the same site. Simple arrows in b2 \& b3 point at clusters of bacteria mobilized to the rock surface upon the application of the KBYO nutrient solution. Full arrows point at fresh deposits of microbial calcite, which are-shortly after completion of the treatment-at an initial state with heterogenous distribution (b2 vs. b3). Comparing b1) with c suggests that microbial calcite crust formation was incomplete prior to exposure and thus susceptible to rapid erosion

\section{Conclusion}

Beam deceleration, an accessory technique that can be combined with standard tungsten cathode SEMs, has been shown to deliver a viable option to study sequential states of surface conditions on non-conductive materials. With a relatively simple modification of the specific sample holder accessory, accommodation of larger samples such as geological rock cores of $25 \mathrm{~mm}$ diameter and up to $15 \mathrm{~mm}$ height with non-polished, rough surfaces was made possible. With respect to the field of cultural heritage, as well as other fields, this offers interesting applications beyond the presented case wherever coating for conductivity is no option due to subsequent treatments, analytical processes with additional techniques, or simply due to the difficulty of achieving a continuous coating on open structures. 
While there have been several studies presenting and discussing images of microbial calcite formation, the presented approach is new in the sense that identical test fields at the micron scale were imaged at different stages of the process, weeks and months apart, including outdoor weathering, to tell the true story. The high sensitivity of the beam deceleration approach allows to contrast bacteria and biofilms on inorganic substrates without the need of sample coating on a standard tungsten cathode SEM.

Visualization of microbial calcite formation that can potentially lead to a consolidating effect upon treatment with KBYO nutrient solution on Barrois-Oolith from Savonnières-enPerthois was realized with sequential images acquired with the beam deceleration technique at different stages of the process by comparing identical sites at the micronscale. In order to achieve a sustainable effect with this method north of the Alps, however, the procedure may require adjustments to the one practiced in southern Europe so that production outweighs erosion of microbial calcite. Documentation with the beam deceleration technique to accompany such treatments will thus greatly help to better understand and actively influence the process for a successful outcome.

Author's contribution Scherrer NC contributed to development of imaging methodology and approach, modification of sample holder, image generation, student coaching. Kocsis M carried out sample preparation, treatment, organization, literature review, and data treatment within the context of his MA Thesis. Dariz P performed polarization microscopy and student coaching. Gervais $\mathrm{C}$ performed project initialization and was MA Thesis referee.

Funding Open access funding provided by Bern University of Applied Sciences.

\section{Declarations}

Conflict of interest This is an independent study with no external funding and no conflict of interest.

Availability of data and material Kbyo is a registered trademark and commercially available (kbyobiological.com). Beam deceleration accessory is available from Zeiss. Modification of the sample holder was produced in-house (HKB).

Code availability Commercially available software SmartSEM (Zeiss) and Heliconfocus (Heliconsoft) has been used without modification.

Open Access This article is licensed under a Creative Commons Attribution 4.0 International License, which permits use, sharing, adaptation, distribution and reproduction in any medium or format, as long as you give appropriate credit to the original author(s) and the source, provide a link to the Creative Commons licence, and indicate if changes were made. The images or other third party material in this article are included in the article's Creative Commons licence, unless indicated otherwise in a credit line to the material. If material is not included in the article's Creative Commons licence and your intended use is not permitted by statutory regulation or exceeds the permitted use, you will need to obtain permission directly from the copyright holder. To view a copy of this licence, visit http://creativecommons.org/licenses/by/4.0/.

\section{References}

1. E. Boquet, A. Boronat, A. Ramos, Nature 246, 527-529 (1973)

2. S. Castanier, G. Le Metayer-Levrel, J.P. Perthuisot, Sed. Geol. 126, 9-23 (1999)

3. W. De Muynck, N. De Belie, W. Verstraete, Ecol. Eng. 36, 118-136 (2010) 
4. N.K. Dhami, M.S. Reddy, A. Mukherjee, Front. Microbiol. 5, 304 (2014)

5. C. Jimenez-Lopez et al., Chemosphere 68, 1929-1936 (2007)

6. C. Rodriguez-Navarro et al., Appl. Environ. Microbiol. 69, 2182-2193 (2003)

7. P. Tiano, L. Biagiotti, G. Mastromei, J. Microbiol. Methods 36, 139-145 (1999)

8. Y. Al-Salloum et al., Constr. Build. Mater. 154, 857-876 (2017)

9. F. Jroundi et al., Nat. Commun. 8, 1-13 (2017)

10. B. Perito et al., J. Cult. Herit. 15, 345-351 (2014)

11. D.W. Phifer, P. Wandrol, T. Vystavel, Microsc. Microanal. 15, 236-237 (2009)

12. J.C. Bouwer et al., Adv. Struct. Chem. Imaging 2, 13 (2016)

13. A. Endo et al., Colloids Surf. A-Physicochem. Eng. Asp. 357, 11-16 (2010)

14. J. Jiruse, M. Havelka, F. Lopour, Ultramicroscopy 146, 27-32 (2014)

15. K. Ohta et al., Micron 43, 612-620 (2012)

16. Kocsis, M., Biomineralisation als Konservierungsmethode für verwitterte, karbonatische Natursteinoberflächen, M. A. -Thesis, Hochschule der Kuenste Bern (Bern University of Applied Sciences, 2020), pp. 284 